\title{
Les années 90
}

\section{COMMUNICATION ET RÉCIT : ÉCHOS D'UNE RELATION TUMULTUEUSE}

\section{Philippe Marion ${ }^{1}$}

Dans sa quête d'identité territoriale et dans ses cheminements interdisciplinaires, la jeune science de la communication ne pouvait ignorer les théories du récit. Le récit comme modèle conceptuel, comme mode d'échange privilégié entre les hommes, comme manière dynamique de représenter le monde et de se définir individuellement et socialement face à lui.

Entre pensée communicationnelle et pensée narratologique, les terrains d'entente sont multiples et les rapprochements historiques ne manquent pas. Ce qui, malheureusement, entraîna aussi quelques malentendus et ambiguités. Le structuralisme et la sémiotique narrative qui s'en inspire ont certes célébré le récit comme objet d'étude, mais ils ont sans doute aussi contribué à répandre un certain

1 Professeur au Département de communication de l'Université catholique de Louvain.

Recherches en communication, $\mathrm{n}^{\circ} 11,(1999)$. 
narratocentrisme dans l'étude de la communication. La structure narrative n'est-elle pas devenue ainsi un modèle aveuglant de communication, jusqu'à se confondre avec elle ?

Il ne faudrait cependant pas réduire la narratologie à un avatar historique de l'histoire des sciences de l'information et de la communication. Les lignes qui suivent tentent de ressaisir brièvement son affleurement «communicationnel» et de présenter l'actualité de sa pertinence. Mieux, en s'ouvrant au champ médiatique et en s'appropriant la pensée herméneutique de Paul Ricœur, la narratologie semble s'offrir de nouvelles perspectives encore largement inexploitées.

\section{Le récit comme bon-objet de la sémiotique structurale}

On sait combien le structuralisme a révolutionné l'étude du récit. Relayé par l'impulsion d'auteurs tels que Greimas, Todorov, Genette Bremond ou Barthes, la pensée structuraliste a consacré la narratologie en tant que nouvelle science littéraire. A côté des géniaux ancêtres tels Platon et Aristote, l'analyse structurale du récit se réclame d'au moins deux pionniers décisifs : Lévi-Strauss et Propp. Dans sa démarche anthropologique, le premier invite à traduire le fatras hétérogène des mythes afin d'en dégager quelques structures universelles permanentes, matrices de tout mythe particulier. Sous la surface bigarrée des narrations mythiques se trouve une grammaire où se combinent un ensemble déterminé de «mythèmes». Tel est le véritable sens du mythe. Parallèlement, on connaît le succès du modèle formaliste de Propp qui, en 1928, propose de réduire les contes merveilleux russes à 31 fonctions fixes et à 7 sphères d'actions.

Greimas et sa suite vont dès lors généraliser ce modèle structural de sorte qu'il s'applique à toutes les formes de récits, voire de discours et de textes. Pour Todorov et Barthes, le récit ne se différencie guère de la langue. Tout récit et chaque épisode de celui-ci se comportent comme une vaste phrase qui combine ses unités de plusieurs façons.

Avec sa sémantique structurale, et son célèbre schéma actanciel, Greimas radicalise l'abstraction -rend plus ascétique- le modèle de 
Propp. Plusieurs auteurs, tels Brémond, le suivront dans le sens d'une abstraction croissante, c'est-à-dire selon une visée d'application plus universelle encore. Le modèle narratif greimassien n'a pas fini de faire parler de lui. Son école l'utilise et le développe constamment en l'étendant à de multiples formes de discours ou de «textes». Ce terme étant à prendre dans une acception très large allant du marketing à la «sémiotique de l'affect», en passant par l'architecture ou l'interprétation des parcours empruntés par les usagers du métro.

Dès sa conception initiale, le «schéma narratif canonique» de Greimas accorde une place importante à la communication. Ainsi entre le destinateur et le sujet-héros s'établit une «structure contractuelle» ${ }^{1}$, le premier «manipulant» l'autre pour mobiliser son désir vers un objet de quête. Dans l'esprit de Greimas, la manipulation se définit en tant que «communication (...) dans laquelle le destinateur-manipulateur pousse le destinataire-manipulé vers une position de manque de liberté (ne pas pouvoir ne pas faire), au point que celui-ci est obligé d'accepter le contrat proposé» ${ }^{2}$. A côté de cette forme "interne" de communication par la manipulation on se rappellera surtout que, par son «contrat», le sujet est placé dans la nécessité d'aller conquérir un objet pour le communiquer au destinataire. Tel est le fameux «axe de la communication» :

Le héros se trouve ainsi placé dans le champ d'une communication, d'un don. En échange, lors de la sanction finale, en contre-don, il (ou l'un des sujets) sera reconnu comme héros ${ }^{3}$.

On s'aperçoit aisément à quel point ce type de schématisation narrative ressemble aux modèles canoniques (linéaires) de la communication. La transmission d'un message d'un émetteur vers un récepteur ressemble à la relation qui se tisse, via l'objet de la quête du héros, entre le destinataire et le destinateur. Cette ressemblance a parfois fini par occulter l'essence narrative du schéma greimassien initial. D'où une série de glissements et de généralisations abusifs : certains pédagogues ou vulgarisateurs pressés n'ont pas hésité à conférer une autonomie quasi universelle à l'axe de la

1 J.-M. ADAM, Le texte narratif, Paris, Nathan, 1985, p. 77.

2 A. Greimas et J. Courtès, Sémiotique, dictionnaire raisonné de la théorie du langage, Paris, Hachette, 1979, p. 220.

3 J.-M. ADAM, op. cit., p. 78. 
communication, alors que celui-ci ne représente, somme toute, qu'un des trois agencements modulaires de la structure actancielle, aux côtés de l'«axe du pouvoir» et surtout de l'«axe du désir». Il s'ensuivit de malheureuses confusions déclinées sous le mode : la communication équivaut au récit. Toute communication repose sur un récit plus ou moins apparent. Mieux, toute communication est récit. Ainsi confondus dans la même structure abstraite, cette perte d'identité de l'un et de l'autre concept a sans doute causé quelque dommage à la pertinence de la narratologie dans l'étude de la communication.

\section{De l'emprise structurale au souci de la relation narrative}

L'influence de la pensée structurale sur la compréhension du récit aura permis de démystifier implacablement celui-ci et de le dissocier de son halo de littéraire subjectivité. Comme tout autre produit du langage, le récit doit être considéré comme une construction dont les mécanismes peuvent être isolés, classés et analysés comme les objets de toute autre science. Le sens du récit ne procède plus d'une expérience individuelle quasi romantique, ni de l'autorité d'une sorte de deus ex machina. Le sens du récit est d'abord la résultante objective de systèmes de signification partagés. Avant de traduire, de transposer dans la fiction ou de refléter la réalité, le récit y génère du sens au sein de sa propre cohérence structurale. Comme le langage. Sur ce point, le structuralisme apporte une rigueur et une démarche dont l'esprit demeure scientifiquement salutaire.

Terry Eagleton souligne à propos de ce constructivisme structural : «le sens n'est plus une expérience privée, ni le fait d'une volonté divine : c'est le produit de certains systèmes de signification partagés. (...) Le structuralisme est l'héritier moderne de cette conviction que la réalité, et notre expérience de la réalité sont en discontinuité». Mais, très sévère, il ajoute : «le structuralisme est $(.$.$) terriblement an-$ historique : les lois de l'esprit qu'il affirme isoler -les parallélismes, les oppositions, les inversions et le reste- se situent à un niveau de 
généralité très éloigné des différences concrètes de l'histoire humaine» 1 .

Ainsi le structuralisme se caractérise par l'exclusion ironique du monde matériel pour éclairer au mieux la conscience que nous en avons. «Pour quiconque croit que la conscience a une dimension pratique importante inséparable de nos façons d'agir dans et sur la réalité, une telle démarche est vouée à l'échec. C'est comme tuer une personne afin d'en examiner plus facilement la circulation sanguine» ${ }^{2}$.

Après l'apogée du «structuralisme» narratif, plusieurs auteurs, pourtant imprégnés de ce courant de pensée, tentèrent de faire progresser la compréhension de cette forme particulière de communication qu'est la narration. Celle-ci étant à prendre dans un sens assez large des «grands choix techniques qui régissent l'organisation de la fiction dans le récit qui l'expose» ${ }^{3}$.

On songe d'emblée à Barthes. En 1970, son $S / Z$ affirme une réelle rupture face à la clôture structurale. Le récit, le texte, doivent être appréhendés dans leur pluriel -le miroitement du sens-que jamais aucune lecture particulière, aussi structurée soit-elle, n'épuise. Comme tout récit est forcément un tressage d'autres récits, d'autres textes, d'autres savoirs, il faut l'investir selon différents regards qu'ils s'appellent «voix» ou «code». Les sédimentations laissées par l'éclairage mêlé de chacun de ceux-ci apporteront leur pierre à l'édifice -la tour de Babel- du sens.

En 1972, avec Figures $I I I^{4}$, Gérard Genette prend quelque distance avec le récit comme manifestation immanente d'une structure invariante pour s'interroger sur les façons «intratextuelles» de gérer le texte narratif. C'est-à-dire sur les manières de répartir, au sein du texte, les rôles des instances porteuses de récit. Les travaux de Genette ont ainsi abordé les dimensions constitutives du «discours narratif». Il propose de réserver le terme de récit à l'ordre des événements au sein du texte seul. L'histoire, de son côté, renvoie à l'organisation (chrono)logique des événements telle que le lecteur peut la

1 T. EAgleton, Critique et théories littéraires. Une introduction, Paris, P.U.F., (trad. fr.), 1994, p. 108.

2 lbid., p. 109.

3 Y. ReUter, L'analyse du récit, Paris, Dunod, coll. «Les topos», 1997, p. 39.

4 G. GenetTe, Figures III, Paris, Éd. du Seuil, 1972. 
reconstituer référentiellement à partir du texte. Vient enfin la narration qu'il limite à l'acte de raconter, c'est-à-dire à l'acte d'énoncer un récit.

Le narrateur genettien peut en outre jouer sur cinq catégories centrales : l'ordre, la durée, la fréquence, l'humeur et la voix. Ces deux dernières catégories méritent quelques explications, vu l'écho (et les controverses) qu'elles trouveront indirectement dans des approches ultérieures. La catégorie de l'humeur, tout d'abord. Genette la subdivise en deux entités : la distance et la perspective. La distance concerne le lien entre la narration et le matériel sémiotique du texte, notamment en reprenant la distinction platonicienne entre le choix de raconter 1'histoire (diégèse) ou de la représenter par imitation (mimèsis). Cette allusion -ici très discrète et presque anecdotiqueapportée aux caractéristiques du média narratif trouvera un développement important dans la nouvelle narratologie médiatique dont il sera question plus loin. La perspective renvoie à la notion de point de vue adopté par le narrateur, notamment à l'égard du mode de relation qu'il entretient avec les personnages. Cette analyse des différentes focalisations narratives sera réinvestie et développée par la suite, notamment par les analystes du cinéma et de la télévision. C'est le cas des travaux sur la focalisation filmique («ocularisation», «auricularisation») menés par Jost. Enfin, avec la catégorie de la voix, Genette se penche sur l'acte de narration, ce qui engage une sorte de typologie du narrateur et du narrataire définis par le texte.

\section{Narrateur cherche narrataire modèle}

Face à la clôture du récit-structure, Genette a donc le mérite de souligner (rappeler) la différence entre la narration comme acte, comme processus de raconter et ce qu'il nomme la narrativité : ce que l'on raconte effectivement. Même dans une autobiographie, le «je» qui raconte semble identique au «je» qui se raconte, mais il en est aussi différent.

En se posant la question des dispositifs et des stratégies présidant à l'acte de raconter, la narratologie devait dès lors s'ouvrir à une dimension pragmatique par ailleurs en plein essor dans les champs de la linguistique et des théories de la communication. Qui énonce le récit ? Comment cet énonciateur imprègne-t-il son énonciation 
narrative ? Comment positionne-t-il son narrataire au sein de son message ? Toutes ces questions trouvent un nouvel intérêt.

En se ralliant à la bannière «dire le monde, c'est se dire», en partageant l'attention théorique portée à la part d'opacité réflexive liée à toute transitivité référentielle, la narratologie se cherche une nouvelle légitimité. Face à la transparence magique du monde raconté, et face aux invariants structuraux que ce monde raconté décline inlassablement, l'attention se porte dès lors sur les instances racontantes saisies dans leur stratégie relationnelle avec une autre instance : le lecteur. Ou plus globalement, le consommateur de récit.

C'est que le récit -on se souvient du fameux «How to do things with words» de Austin- réalise quelque chose dans son énonciation. Il s'agit donc de se pencher sur la visée fondamentalement performative du récit. On gère certaines conventions -et une certaine intertextualité, ajoutera Kristeva- pour chercher à produire certains effets sur le lecteur.

D'un point de vue historique - du moins en ce qui concerne la narratologie française- l'attention portée au partenaire de la relation narrative apparaît assez tardive face aux travaux visionnaires de Mikhail Bakhtine. Dès 1929, celui-ci se défiait à la fois de l'objectivisme saussurien et du subjectivisme des lectures où le texte n'est que prétexte à états d'âme, à paraphrases affectives. Pour lui, le langage et forcément le récit ne peuvent se comprendre que dans leur essence dialogique, inévitablement tournée vers l'autre. Si Bakhtine accepte une certaine autonomie du langage, il souligne aussi à quel point ce langage s'englue dans des relations sociales définies ${ }^{1}$. Et celles-ci, à leur tour, n'ont de sens que dans un système socio-économique et idéologique plus large.

S'interroger sur la signification du signe? Soit, mais cela demeure insuffisant. On ne peut faire l'impasse sur son histoire complexe que des groupes sociaux, des classes, des individus et des discours cherchent à s'approprier et à imprégner de leurs propres significations. En bref, pour Bakhtine, langue et récits n'ont de signification que dans un processus de dialogues sociaux souvent mêlés de conflits idéologiques. Cette façon de voir sera partagée, quelques décennies plus tard, par un Lafaye. 1 Voir notamment M. BAKHTine, Esthétique et théorie du roman, Paris, Gallimard,
1978. 
Le souci du partenariat narratif a trouvé une modélisation très élaborée sous l'impulsion d'auteurs tels Umberto Eco et autres tenants de ce qu'on appelle parfois la «théorie narratologique de l'interprétation coopérante»1. Le «lecteur modèle»: la notion est désormais célèbre... Ce lecteur modèle tient à sa disposition tous les codes susceptibles de révéler le récit dans l'intégralité de son intelligibilité. Il est, selon Eco, le coopérateur idéal, capable de saisir toutes les visées intertextuelles du récit. Mieux, d'en saisir et d'en exploiter des germes insoupçonnés. Il doit être pourvu de cette compétence cognitive essentielle au déchiffrage du récit, être inattaquable dans l'application de ce savoir compétent et dégagé de toute restriction invalidante. Décrire un récit suppose donc la description du narrataire modèle tel que le texte narratif le construit en fonction du positionnement solidaire d'un narrateur tout aussi modèle. En cela, la théorie du lecteur modèle repose sur une exploitation optimale (idéalisée) des traces de l'énonciation (plus précisément de l'énonciataire) dans le message, principe sur lequel se fonde, en partie, la sémiopragmatique.

Pressentant le reproche d'un nouveau formalisme qui viendrait d'emblée figer l'ouverture communicationnelle de la narratologie, Eco se défend avec insistance. Pour lui, le lecteur modèle est une fiction exploratoire, heuristique, une cristallisation modélisée de toute la compétence mobilisée pour la lecture. Il reste néanmoins que peu de sujets réels risquent de correspondre totalement à ces exigences. En se sens, un texte peut-il réellement prévoir son avenir d'interprétation? N'en déplaise à Bakhtine ou à Eco, le récit ne peut pas contrôler et délimiter ses lectures comme nous le faisons ou comme nous tentons de le faire dans une conversation face à face, ou dans le cadre d'une interaction interpersonnelle réelle. Pour reprendre encore les paroles d'Eagleton: «1'“anonymité" (de l'œuvre) fait partie de sa structure et n'est pas un accident malheureux» ${ }^{2}$. Poussé à son extrême, le modèle du lecteur modèle, crée une sorte de lecteur sans état, sans classe, asexué, dégagé de toutes caractéristiques ethniques et sans aucun postulat culturel limitant. Un tel super-lecteur n'est rien moins

1 Selon Eco, l'activité du lecteur modèle se concentre sur une coopération interprétative adéquate des consignes. U. ECO, Lector in fabula, Paris, Grasset, 1979.

2 T. EAGLETON, op. cit., p. 119. 
qu'un «sujet transcendantal libre de tous déterminants sociaux limitatifs»1.

Avec un peu de recul, un point important est généralement partagé, marquant un détachement décisif vis-à-vis de la clôture structuraliste et l'ouverture au souci de la réception : «le récit n'est plus, dorénavant, un énoncé inerte. (...) Il doit aussi être envisagé dans la relation que sollicite son actualisation par l'énonciation narrative. Car lorsque l'on échange de l'information par la voie du récit, on se place dans un cadre, dans un système codé. Un système où se tresse un réseau d'instructions et d'attentes cristallisant le jeu complexe entre narrateur et narrataire ${ }^{2}$. Les partenaires de la communication narrative acceptent donc d'honorer respectivement les termes d'un contrat ou d'une promesse. Le raconteur tente de gérer les «horizons d'attente» ${ }^{3}$ de celui auquel il s'adresse, celui-ci essaye -est supposé essayer- d'interpréter les instructions qu'il croit déceler dans ce qu'il reçoit.

\section{La narratologie et ses nouveaux horizons médiatiques}

Après l'euphorie narrativisante d'un certain structuralisme narratif, après les critiques qui ont suivi, après les ouvertures stimulantes sur les consommateurs de récit par les théories de la réception, la narratologie connaît depuis quelques années d'autres développements. Ceux-ci permettent d'entrevoir de nouvelles interactions avec les sciences de la communication. Trois ancrages théoriques différents mais solidaires me semblent déterminants pour fonder la pertinence d'un nouvel espace narratologique au sein de la communication : la narratologie comparée dont la confrontation avec différents systèmes sémio-médiatiques engrange de précieuses sédimentations théoriques, les conséquences narratologiques de la

1 Ibid., p. 120.

$2 \mathrm{Ph}$. MARION, "Le récit médiatique comme modèle d'interprétation", in G. DERÈze (coord.), La quatrième mi-temps, Louvain-la-Neuve, Observatoire du récit médiatique (ORM), 1995, pp. 23-40.

3 Pour cette notion d'horizon d'attente, on peut se référer aux théoriciens de la réception littéraire et surtout à W. ISER, "La fiction en effet", Poétique, ${ }^{\circ} 39$, sept. 1976, p. 279. 
pensée philosophique de Paul Ricœur, et enfin la conceptualisation problématique du récit médiatique.

A l'époque où l'analyse structurale du récit battait son plein, plusieurs auteurs, tels Barthes, exprimait la nécessité d'étendre le modèle de la «langue du récit» à d'autres systèmes sémio-narratifs : la bande dessinée, la presse, le théâtre, le cinéma... Après les avancées

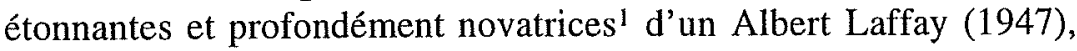
l'analyse du récit filmique trouve en Christian Metz un point d'ancrage reconnu et célébré. Si son approche du film est d'abord, du moins à l'époque de la «grande syntagmatique », directement inspirée de la linguistique structurale, elle n'en manifeste pas moins une certaine attention à la singularité expressive du cinéma au sein des arts narratifs. Ne se plaisait-il pas à rappeler combien le film se caractérise par une narrativité bien «chevillée au corps» ${ }^{2} \ldots$

A côté d'une première vague d'explorations des différents médias narratifs où l'on recherche surtout l'organisation des invariants structuraux sans trop se soucier du potentiel propre des différents médias visités, un nombre croissant de recherches en viennent à prolonger la pensée de Metz. On s'interroge dès lors sur la manière dont le récit négocie avec le média qui le transmet. S'inspirant des concepts antiques de diégèsis et de mimèsis, André Gaudreault se penche sur la problématique complexe qui sous-tend le passage du récit littéraire au récit filmique, non sans envisager la question du récit scénique. Chacun à leur façon et à des degrés divers, des auteurs tels que Altman, Bordwell, Odin, Vanoye, Gardies, Château et d'autres interrogent l'expression ou l'institution cinématographiques quant à leur capacité plus ou moins spécifique de «produire» du récit. Un même souci comparatiste anime beaucoup de recherches portant sur la narration dans d'autres médias tels la télévision, avec notamment les travaux de Jost, Verón, Lochard, Soulages ou Nel.

L'intérêt de ces explorations médiatiques ne réside pas dans l'attention, excessive parfois, apportée à l'inventaire des singularités des différents médias narratifs. Car on risquerait ainsi de remplacer la rigidité du modèle de la structure narrative par une rigidité tout aussi abrupte : celle du modèle des «invariants» médiatiques manifestant une sorte de dérive ontologique... L'intérêt, donc, est ailleurs : il

1 A. LAFFAY, Logique du cinéma, Paris, Masson, 1964.

2 C. MeTZ, Essais sur la signification au cinéma, Paris, Méridiens Klincksieck, p. 52. 
s'agit d'appréhender la narration dans son interdépendance complexe, instable, évolutive avec le potentiel du média qui l'énonce. C'est dans cet esprit que Gaudreault invite à comprendre le récit filmique comme la rencontre d'une narrativité intrinsèque (celle du média filmique) et d'une narrativité extrinsèque. C'est dans le même esprit que l'on peut suggérer, plus globalement, l'avènement d'une «médiatique narrative» (ou d'une narratologie médiatique), la démarche consistant à étudier l'interaction de la médiativité, d'une part et de la narrativité, d'autre part. La médiativité -la médialité- «rassemble tous les paramètres qui définissent le potentiel expressif et communicationnel développé par le média. Elle est donc cette capacité propre de représenter -et de placer cette représentation dans une dynamique communicationnelle-qu'un média possède ${ }^{1}$. Cet ensemble est luimême régi par une contingence décisive : les discursivités et les usages sociaux de ces médias qui -variant selon les époques et les contextes- influencent radicalement leur définition et la font évoluer.

La prise en compte de cette interaction du média et du récit ouvre des espaces de recherches féconds pour la narratologie comparée qui, elle-même, constitue un maillon indispensable à l'épanouissement d'une véritable narratologie médiatique. S'ouvrent alors les territoires prometteurs des lectures transsémiotiques, transmédiatiques, intermédiales. Territoires d'autant plus fertiles dès lors qu'on les expose, malgré une complexité croissante, à d'autres facteurs de démultiplication, notamment le vecteur générique. Car les genres charrient leur propre pacte -ou promesse- de communication implicite, et donc leurs «horizons d'attente» qui, à leur tour, épousent ceux de la narration et ceux du média. Concernant la télévision, par exemple, John Corner a raison de nous mettre en garde contre la généralisation abusive que manifeste une idée aussi répandue que celle du réalisme télévisuel. Le pluriel est indispensable, insiste-t-il, et on ne peut parler que des réalismes télévisuels liés à la pluralité générique : «Les attentes des spectateurs, leurs niveaux d'attention, les cadres cognitifs et affectifs qu'ils assignent aux émissions varient en fonction des genres» ${ }^{2}$.

I Ph. MARION, « Narratologie médiatique et médiagénie des récits », Recherches en communication, $\mathrm{n}^{\circ} 7,1997$, p. 79.

2 J. CORNER, «Genres télévisuels et réception », Hermès, n 11-12, p. 118. 


\section{L'ouverture ricourienne}

Dans le cadre de sa réflexion herméneutique, Paul Ricour consacre une part considérable au récit qu'il pose dans son indéfectible rapport au temps. On n'a sans doute pas encore mesuré toute la richesse que cette remise en question exogène -elle vient d'un philosophe- mais décisive de la narratologie, dans la perspective stimulante de ce rapport entre temps et récit.

Avec Ricour, soulignent Adam et Revaz, «le texte narratif est définitivement libéré de la clôture structurale» ${ }^{1}$. Le philosophe insiste en effet sur l'importance d'une conception génétique qui précisément s'oppose au structuralisme :

Le point de vue structural est ainsi globalement opposé au point de vue génétique. Il cumule à la fois l'idée de synchronie (priorité de l'état de langue sur l'histoire), l'idée d'organisme (la langue comme unité de globalité enveloppant les parties), enfin l'idée de combinatoire (la langue comme un ordre fini d'unités discrètes) $)^{2}$.

Ricœur en vient alors à dénoncer le refus du structuralisme de prendre en compte la force pourtant décisive de l'historicité en subordonnant indûment la diachronie à la synchronie :

Mais alors que l'explication structurale paraît à peu près sans reste lorsque la synchronie l'emporta sur la diachronie, elle ne fournit qu'une espèce de squelette, dont le caractère abstrait est manifeste, lorsqu'il s'agit d'un contenu surdéterminé qui ne cesse de donner à penser et qui ne s'explicite que dans la suite des reprises qui lui confèrent à la fois interprétation et rénovation ${ }^{3}$.

Il faut donc, poursuit Ricour restaurer l'intelligibilité du changement, car il n'est pas d'identité narrative sans dynamique évolutive. C'est dans cet esprit qu'il faut recadrer le cycle ternaire d'interprétation des trois mimèsis, modèle qui a acquis aujourd'hui une notoriété légitime. Rappelons-en brièvement le principe.

1 J.-M. Adam, Fr. Revaz, L'analyse des récits, Paris, Éd. du Seuil, coll. «Mémo», p. 11.

2 P. Ricceur, Temps et récit, tome 2, La configuration du temps dans le récit de fiction, Paris, Éd. du Seuil, 1984.

3 P. RICEUR, Le conflit des interprêtations. Essais d'herméneutique I, Paris, Éd. du Seuil, 1969, p. 53. 
La première mimèsis prend en compte le temps humain vécu et le sens de «l'agir humain» qui nourrissent la production de tout récit. Car «la composition de l'intrigue est enracinée dans une précompré-

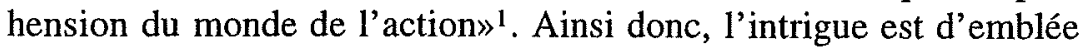
définie comme la mimèsis d'une action.

La deuxième mimèsis recouvre le plan du récit à proprement parler : elle repose sur l'acte de raconter ou de re-présenter, de configurer le temps humain. L'étape est décisive : la succession d'actions se débarrasse de son hétérogénéité, elle est organisée, finalisée, vectorisée dans et par la visée homogénéisante assurée par un acte configurant dans le temps que Ricœur nomme : «mise-enintrigue». Cette étape de la cristallisation en récit nous offre l'opportunité d'un exercice de maîtrise du temps et de l'action : «Je vois dans les intrigues que nous inventons le moyen privilégié par lequel nous re-configurons notre expérience temporelle confuse, informe et, à la limite, muette» ${ }^{2}$.

Enfin, le plan de la refiguration ou mimèsis III prend en considération la réception du récit. En s'appropriant le récit, le récepteur reconfigure le monde / temps mis en intrigue au cours de la mimèsis II.

Comprendre un récit, c'est donc comprendre les actions qui y sont représentées et temporalisées. Selon Bertrand Gervais, «cela requiert une compétence particulière qui est la pré-compréhension de l'action. Le passage de la mimèsis I à la mimèsis III, par le biais de la mimèsis II, qui est le récit comme mise en intrigue et représentation discursive d'action, n'est possible que parce que toutes ont en commun cette même précompréhension ${ }^{3}$. Si nos actions et nos représentations d'actions -le vécu humain- sont le terreau du récit, notre vécu est, en retour, imprégné et transformé par les récits auxquels nous sommes exposés. «Le temps, précise Ricœur, devient humain dans la mesure où il est articulé de manière narrative; en retour, le récit est significatif dans la mesure où il dessine les traits de l'expérience temporelle» ${ }^{4}$.

On voit comment s'enchevêtrent et s'interfécondent ces trois mimèsis. Elles ne constituent nullement des plans indépendants ou

1 P. RICceur, Temps et récit, tome I, Paris, Éd. du Seuil, 1983, p. 100.

Ibid., p. 13.

3 B. GervaIs, Récit et actions. Pour une théorie de la lecture, Québec, Les Éditions du Préambule, 1990, pp. 15-16.

4 P. RICEUR, Temps et récit, tome I, op. cit., p. 17. 
distincts. Elles tressent un cycle, une boucle qui, telle une spirale, génère un mouvement perpétuel. $\mathrm{Et}$, de récits en récits, en déposant sans relâche ses sédimentations, ce mouvement triadique sculpte une identité narrative.

Les enjeux de ce procès cyclique de l'identité narrative sont nombreux et importants. En premier lieu, ils permettent de penser les rapports de la narratologie et de la communication sur une base plus féconde. Car «bien des savoirs sociaux brouillent les savoirs narratologiques» ${ }^{1}$. A cette mise en garde, le modèle de Ricœur offre une excellente parade. En effet, cette définition de la triple mimèsis rend compte de l'extension totale du récit, de ses possibilités de production aux modalités de sa réception. Et la narratologie ne doit précisément pas s'enfermer dans une mimèsis II qui serait par là injustement dissociée du mouvement triadique ; ce qui n'empêche pas de considérer cette mimèsis II comme le point d'appui principal de la narratologie, sur un plan méthodologique. Si l'on prend la peine d'interpréter les récits selon la première et troisième mimèsis, les «brouillages sociaux» évoqués ci-dessus s'intègrent en fait dans les dimensions de la production et surtout de la réception narratives. Assimilés de la sorte, les brouillages ne constituent plus un bruit de la communication narrative mais bel et bien une condition d'existence.

En outre, Ricœur construit un important dialogue entre l'aspect «quasi historique de la fiction» et l'aspect «quasi fictif du passé historique». Selon Olivier Mongin ${ }^{2}$, cette approche ricœurienne permet de comprendre «de quelle façon unique en son genre, l'imaginaire s'incorpore à la visée de l'avoir été, sans en affaiblir la visée réaliste». S'ouvre alors l'opportunité de recadrer la question plus globale du rapport factuel/fictionnel telle qu'elle se pose notamment dans l'étude des récits médiatiques.

\section{Des trois mimèsis aux récits médiatiques}

La boucle mimétique de Ricœur offre donc une ampleur nouvelle à la narration. La dimension communicationnelle des récits y dépasse non seulement la clôture structurale, elle confère une épaisseur

1 Fr. Jost, «Quand y a-t-il énonciation télévisuelle ?», in Penser la télévision, Actes du colloque de Cerisy, Paris, INA-Nathan, 1998, p. 37.

2 O. MongIN, "Face à l'éclipse du récit", Esprit, nº 8-9, août-sept. 1986. 


\section{Le récit médiatique comme laboratoire de la communication?}

Une fois élargi aux récits médiatiques qui rythment la vie d'une collectivité et tressent son imaginaire, le modèle ricœurien initial permet au moins un double programme d'interprétation. Sur le plan narratologique, les récits affleurant dans les médias -et notamment les récits d'information et d'actualité- doivent être analysés dans leur paraître médiatique (mimèsis II). Ce qui renvoie aux multiples aspects -formels, thématiques, sémantiques, génériques, communicationnels...-des mises en intrigue interagissant avec des mises en signes et des mises en média (cf. supra).

Mais, dans sa phase d'extension, le sens de cette configuration doit impérativement être revu selon des déploiements solidaires. En amont, tout d'abord, il faut tenter de saisir ce que les récits retirent d'une précompréhension de l'agir humain, d'une pré-représentation de l'action et du temps. En termes plus concrets, il s'agit d'appréhender le contexte, les conditions de production, l'état d'une socioculture, voire même s'imprégner de l' «air du temps»... Toutes ces composantes tissent une sorte de pré-intrigue ; c'est le terreau narratif préexistant propre à la mimèsis I. Par la vertu de la boucle mimétique, celle-ci se trouve sans cesse nourrie et donc modifiée : les mentalités qui délimitent les faisceaux de déterminations des productions futures évoluent elles aussi selon les récits assimilés et partagés en phase de mimèsis III. Ceci rend quasi nécessaire une histoire du quotidien prémédiatique sans cesse tenue à jour. En aval, il faut réciproquement s'enquérir de la manière dont les récits sont reçus et consommés. Les récits de réception ${ }^{1}$ et les récits circulant à propos des récits médiatiques prennent ici toute leur importance. Soit par exemple, en ce qui concerne le récit journalistique : enquêtes en rédaction, analyse des courriers de lecteurs, et autres enquêtes de terrain pour observer la

1 Parallèlement aux récits médiatiques qu'ils véhiculent, il faut donc aussi percevoir la manière dont les médias sont apprivoisés par les consommateurs qui intègrent les objets domestico-médiatiques dans le récit vécu de leur vie quotidienne. Tel serait l'objet d'une «ethnonarratologie». Celle-ci se propose d'appréhender une «démultiplication» narrative articulée sur trois plans : celui du récit véhiculé par le média, celui du média interagissant avec la réception des récits qu'il médiatise, celui enfin du média tel qu'il s'intègre dans l'histoire (la vie) quotidienne de son consommateur. 
manière dont les récits d'actualité prennent corps et imprègnent une collectivité donnée.

Sur un plan méthodologique (épistémologique), le modèle peut donner lieu à une répartition des tâches : chaque pôle de mimèsis appelle ainsi une autonomisation (inter)disciplinaire. A la mimèsis I correspondent des approches telles que la socio-économie et l'histoire des médias. La narratologie restreinte et élargie -la narratologie médiatique- se porte sur l'analyse des discours narratifs construits en phase de mimèsis II. Quant à la mimèsis III, elle mobilise des études de réception (telles la sociosémiotique, la sociologie de la réception ou ce que nous avons proposé d'appeler l'ethnonarratologie).

En outre, le récit médiatique permet un dialogue transdisciplinaire permanent entre ces orientations de recherche. Chaque pôle a des comptes à rendre aux autres... S'ils gèrent une certaine autonomie dans leur domaine respectif, ils doivent observer une certaine "loyauté fédérale" placée sous le signe d'un objectif commun : la compréhension de l'identité médiatico-narrative saisie dans la complexité de son évolution constante.

Le récit médiatique me semble dès lors présenter un réel intérêt pour la recherche en communication. En tant que projet théorique et conceptuel, tout d'abord, le récit médiatique reproduit dans l'espace limité qui est le sien la complexité et la dynamique interdisciplinaire qui se trouve au cœur de la recherche en communication. Certes, il faut se garder de reproduire l'aveuglement de l'équivalence par amalgame : récit médiatique et communication demeurent résolument différents, mais l'hypothèse du récit médiatique comme métaphore limitée mais révélatrice -un laboratoire ?- de la communication gagnerait néanmoins à être exploitée. Ensuite, comme domaine d'étude malgré tout plus cerné, mieux balisé, le récit médiatique offre à la communication l'opportunité d'une cristallisation, d'une aspérité, d'une réduction salutaire -didactique, heuristique ?- de son objet. Expliquons-nous : on sait combien la communication est un champ complexe et mouvant, au carrefour souvent inconfortable de plusieurs disciplines plus anciennes et, partant, mieux stabilisées. S'il relève pleinement du questionnement ouvert par les études de communication, le champ couvert par le récit médiatique n'en est pas moins plus réduit, plus maîtrisable. Pour éviter le risque réel de dissolution, d'évanescence, la discipline communicationnelle a tout intérêt à se ménager de telles zones d'aspérité qui lui offrent l'opportunité de se 
laisser saisir plus fermement selon un éclairage spécifique. Le récit médiatique constituerait ainsi une aspérité privilégiée pour réinvestir et canaliser l'étoilement transdisciplinaire de la recherche en communication. Tout en demeurant une façon décisive de communiquer. Le récit médiatique est peut-être alors une excellente initiation à la communication comme domaine de recherche. 